\title{
Hyperspectral Imaging for Raw Material Sorting and Processed Product Quality Control
}

\author{
Pilar-Beatriz García-Allende, Olga M. Conde \\ and José Miguel López-Higuera \\ University of Cantabria \\ Spain
}

\section{Introduction}

Agricultural and industrial sectors are more competitive and quality conscious than ever before. To be profitable, industry requires equipment that would ensure pure, high-quality production, and efficient work and cost. This fact is even more important in developed countries to aid companies to defend their position and competitiveness against others where labour cost does not account for such a significant part of the overall manufacturing cost. Raw material sorters, conveyors and processing systems easy to install, energy efficient, reliable, low-maintenance and simple to adjust and control are sought. Even the inclusion of network connectivity capabilities for remote monitoring and communication is also desirable.

Optical Spectroscopy (OS) becomes highly appropriate for this kind of applications because it covers all types of qualitative and quantitative analytical methods based on the interaction of light with living and non-living matter (Schmidt, 2005). For more than 200 years it has been utilized in various fields of science, industry and medicine, particularly in (bio-) chemistry, biology, physics and astronomy. OS is highly specific since each substance is discernible from all others by its spectral properties. In addition, the requirements of the samples are not particularly restrictive. Measurements of different optical parameters as a function of wavelength/energy ("spectrum") provide valuable insights that are not, or not readily, attainable by other analytical methods. Traditional OS techniques generate information on the bulk properties of a sample or a portion taken from it (Millar, 2008). However, there are many aspects of sample properties which result from heterogeneity in composition and the monitoring of this aspect of quality is considerably more challenging. In the last few years Hyperspectral Imaging Spectroscopy (HIS) that integrates conventional imaging and moderate resolution spectroscopy, which was primarily developed for applications in remote sensing and astronomy, has been developed for laboratory use and it has even slowly transitioned into other areas as life sciences or industrial production. The key difference is that, in this technique, entire spectra are collected for each pixel within the image so its advantages in agricultural and industrial procedures such as material discrimination are obvious, i.e. reduced measurement time due to the simultaneous acquisition without the need for scanning mechanics. By assessing specific spectral features at each pixel corresponding to a material point, or by using calibration to quantify 
individual components, an optical parameter map of the material may be generated. There exists a wide variety of systems to obtain this both spatial and spectral information from an object, the so-called imaging spectrometers that differ in their acquisition methods (Aikio, 2001). A review of the existing methods and technologies is performed and compared in terms of their suitability for on-line quality applications in agricultural and industrial environments.

In quality assurance systems the final output should be delivered in a straightforward way facilitating its application, i.e. they have to provide a clear added value to the user (usability concept). For instance, in raw material discrimination, only the presence and position of spurious materials are of interest. No further information is required to determine which jets of pressurized air need to be activated to blow the unwanted material into a container, while the rest of the material continues to another conveyor. Imaging spectrometers collect, however, underpinning information about material optical properties. Therefore, to obtain efficient quality indicators, considerable data management and analysis is required to convert this huge information into the desired operative indicators. The investigation into novel and time efficient compression and interpretation techniques, suitable for laboratory use as well as for real time production lines in industrial sectors, is addressed in this chapter. To this end, Section 2 describes the principles and different approaches for HIS operation. The implementation of the sensor system is provided in Section 3, whereas Section 4 is focused in how the HIS processing unit is structured. Section 5 discusses different classifiers for automatic material sorting. Section 6 presents the results obtained with each proposed alternative comparing their performances in terms of classification accuracy as well as time and space complexity. Finally, some conclusions and future lines of work are outlined.

\section{HIS applied to industry processes}

Raw material on-line characterization and discrimination processes are a key issue in several agricultural (Park, 2004; Xin, 2005) and industrial sectors (Reich, 2008; Herrala, 1997; GarciaAllende, 2008c). Technologies for quality evaluation detect physical properties which correlate well with certain quality factors of the products such as density, firmness, vibrational characteristics, X-ray and gamma ray transmission, optical reflectance and transmission, electrical properties and nuclear magnetic resonance. Each technique has advantages and limitations, such as image resolution, imaging duration, safe handling, sample-specific requirements and availability of details such as surface color, texture, and internal details.

- X-rays, due to their high energy, can penetrate many objects (Mesina, 2007; Kotwaliwale, 2007). However, there is variability in penetration through different materials depending on their properties. Although X-rays great potential, the associated high equipment cost and safety issues make them less practical for on-line applications.

- Colorimetric analysis based on cameras and machine vision are another option (Abbasgholipour, 2010) but they are constrained to characterize the outer layer of the material with high probability of confusing different materials presenting similar colours or due to problems derived from variations on the illumination.

- Optical spectroscopy, based on diffuse reflectance measurements, is another promising method able to extract the chemical composition from the spectrum of the material, which can be employed for sorting. 
- Thermal imaging (Varith, 2003) measures infrared energy emitted from the material. The detected energy is converted into a thermal map called a thermogram. Quality indicators are derived from temperature differences on material points. In some cases, thermal imaging does not require an illumination source.

- $\quad$ LIBS (Laser Induced Breakdown Spectroscopy) determines the chemical composition of laser evaporated material on surfaces (Wiggenhauser, 1998). When material is vaporized by a high energy laser pulse, the composition elements emit their specific fluorescence. This radiation is used to identify them in the evaporated material by spectroscopic methods (Solo-Gabriele, 2004).

Among all the previous techniques, the non-destructive and non-contact characteristics of optical techniques, along with the low equipment cost and absence of material preprocessing, make them a perfect candidate for material characterization and sorting. Imaging spectroscopy measures the optical spectrum of interactions of light and matter (absorption, scattering, reflectance, etc.) and the spatial location of an object in a surface (Lu, 1999). This ability offers a great potential for identifying raw materials while they are transported by the conveyor belt in production plants. Analysis systems can be easily installed over the conveyor belt with minimum interference in the industrial set-up. As a result, work in this area has gained prominence in the last few years and it is being adopted for product development and control of a wide variety of industrial processes (Bonifazi \& Serranti, 2006; Bonifazi, 2008; Gomez-Sanchis, 2008; Leitner, 2009).

Usually, raw materials need to be classified on different quality levels. Present experience has been acquired working for a tobacco industry located in the region of Cantabria (Spain). Apart from the company interest in tobacco sorting procedures, few studies were found about the spectral characterization of tobacco chemical components (nicotine, polyphenols, etc.) (McClure, 1997). Even less work is reported in open literature regarding tobacco quality control in industry which is, at present, mainly performed by time-consuming machine vision techniques. On account of this, it was definitely a real problem-oriented niche for both knowledge and technology production. Moreover, the developed strategies could undeniably be extended to other industrial fields and materials.

The problem to be solved in the tobacco industry consists in the classification of the raw material at the entrance of the production plant. Tobacco leaves are intermingled with a great variety of materials such as plastics, cords, cardboard, papers, etc., mainly due to the manual harvest procedure. These spurious elements or "defects" have to be rejected before starting the process of making cigars. At first, the leaves undergo a mechanical classification procedure, where rejection due to differences of weight, size and density is performed. However, some of the undesired materials go through this coarse classification and additional discrimination systems are required. The design of a completely non-intrusive, non-contact and real-time system capable of performing this identification task, employing an imaging spectroscopic technique, is the aim of this research.

\subsection{HIS basics}

Diffuse reflectance is the most appropriate light-matter interaction for material sorting. It is produced when an incident radiation penetrates the material sample. Once this radiation travels through the sample, it becomes partially absorbed and partially scattered. At the end, some radiation gets out of the material on arbitrary directions. The spectrum of this reflected radiation has now imprinted the effects of the chemical composition of the sample. 
The HIS concept is applied over this parameter. The diffuse reflectance measurement generates a three-dimensional data set, datacube, defined by spatial ( $x$ and $y$ ) and wavelength $(\lambda)$ coordinates (Willoughby et al., 1996; Green et al., 1998). Figure 1 depicts the datacube consisting of the light reflected by the material shown at the foremost part of the cube. Hyperspectral images and the associated reflectance spectra of the vertical lines located at $x_{1}$ and $x_{2}$ positions are shown at the lower part of Fig. 1 . The discontinuous lines highlight the correlation between the real and spectral images of each segment. A colour gradation scale is employed to represent the spectra of each spatial position $y_{i}$ of the vertical lines. When the colour of a pixel is close to red, it means that the intensity of the reflected radiation at that particular wavelength is high. On the other hand, if the pixel approaches blue, the material shows low reflectance.

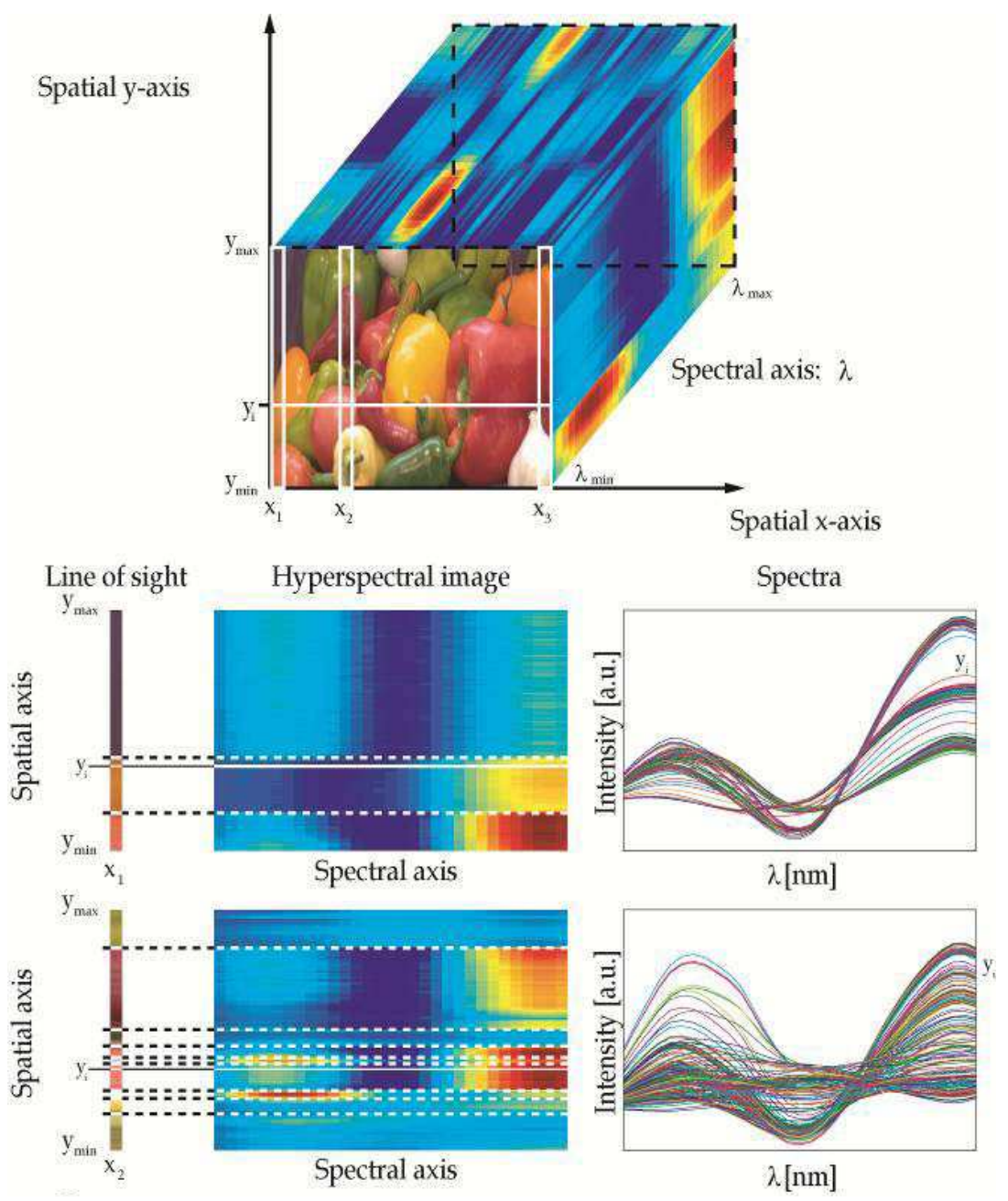

Fig. 1. HIS concept showing how the spectrum of a spatial point is recovered from an image 


\subsection{HIS alternatives}

The datacube provided by imaging spectrometers can be classified depending on the dataacquisition mode. The most conventional types are shown in Figure 2:

- Non-imaging spectrometers are called whiskbroom scanners because they provide the spectrum of a single point within the image, i.e. a column within the image cube. The recording of the whole image implies a double spatial scanning step, and, either scanning mechanics or several single point instruments are, therefore, required. These approaches are respectively time-consuming and expensive.

- Staring imagers, as filtered cameras or tuned wavelength illumination source, electronically scan the spectral domain. The image is collected one spectral plane at a time. Although easy to implement by means of tuneable filters or LED's activation, this configuration has a major drawback. The spectra for each pixel are not taken simultaneously, and, consequently, pixel-to-pixel spectral mixing could occur if the scene is moving while the spectra are being scanned (Willoughby, 1996).

- Pushbroom scanner employs an array of detectors to simultaneously scan the spectral dimension and one spatial dimension. This acquisition mode allows data collection in hundreds or thousands of spectral bands (hyperspectral) to produce an image. This makes a difference respect to colour cameras that collect information in the three red, green, and blue bands or multispectral imaging achieved by tuneable filters. In this way, not only spectral mixing is prevented, but also sensitivity to minor components is improved. There still remains the scanning of the other spatial dimension, but this can be easily achieved by the relative motion between the scene and the imaging spectrometer field of view. As a result, this configuration is particularly suitable for industrial applications, which exhibit a predominance of conveyor belt systems.

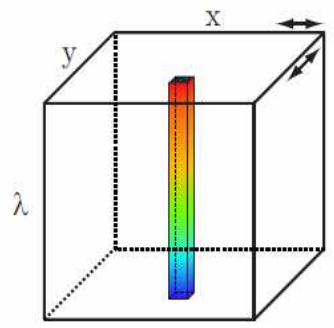

Whiskbroom spectrometer

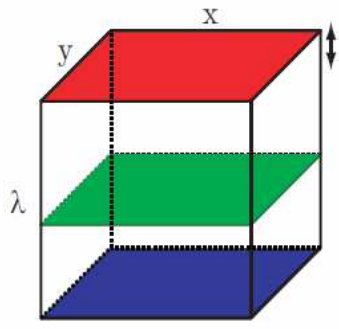

Filtered camera

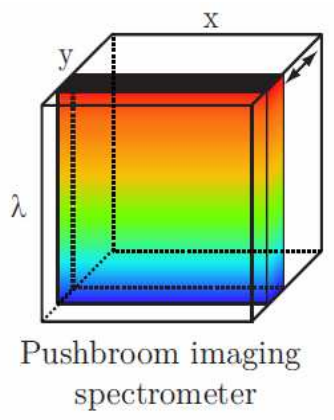

Fig. 2. Spectral imaging sensors depending of their data acquisition modes

Different imaging spectrometers designs can employed depending on the selected dispersive configuration (Aikio,2001). Among the transmissive configurations, imaging spectrometers could be based on PGP (prism-grating-prism) devices, HoloSpec design or Fourier-Transform configurations. On the reflective side, Offner configurations, Echelle gratings, Czerny-Turner layouts and Hadamard transform approaches can be employed.

\section{HIS acquisition and system approach}

A non-destructive, non-contact and real-time optoelectronic system based on a PGP (prismgrating-prism) imaging spectrograph was designed. Figure 3 shows its block diagram. HIS 
images are first recorded by a camera through a PGP device in the "hyperspectral image acquisition" unit, where spectra of the diffuse reflectance of materials are obtained.

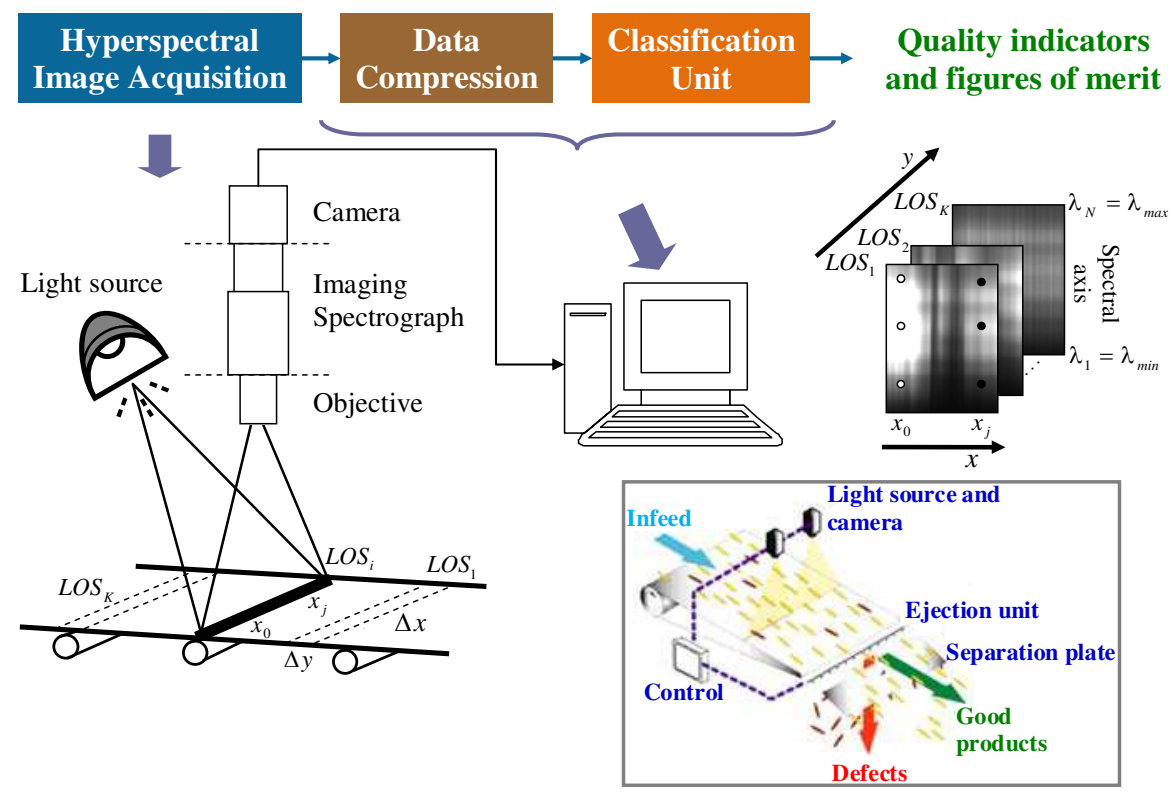

Fig. 3. HIS system for material sorting in industrial processes. Inset: schematic implementation for on-line sorting

In the final set-up, the commercial imaging spectrograph ImSpector (Specim Ltd.) was used. It is a pushbroom scanner that images the spectral dimension and one of the spatial dimensions. The temporal displacement of the sample provides the imaging of the second spatial dimension. The light source consists of two halogen floodlights with a power of 500 $\mathrm{W}$ each. The diffuse reflectance from the material surface is collected by a Navitar Zoom 7000 lens that delivers it into the entrance slit of the imaging spectrograph. This slit fixes the instantaneous "field of view" of the system in the spatial direction to a length $\Delta x$ and a width $\Delta y$. This is the so-called observation line (LOS, Line Of Sight). Each point $x_{i}$ of the observation line has its specific image on the entrance slit. The radiation at the slit is then collimated by a lens and then dispersed whose output direction depends on the wavelength of the radiation. The dispersive element of ImSpector is a volume type holographic transmission grating. This grating is used in a PGP-structure, providing high-diffraction efficiency and good spectral linearity (Vaarala, 1997). Material analysis was performed in the Vis-NIR (Visible-Near Infrared) and NIR (Near Infrared) ranges. Imspector devices V10E $(400-1000 \mathrm{~nm})$ and N24E $(1000-2400 \mathrm{~nm})$ were employed. Dispersed radiation is then focused into the detector plane of a CCD or a CMOS sensor. Every point $x_{i}$ is represented by a line of monochromatic points that represents a continuous spectrum in the direction of the spectral axis. In the Vis-NIR range, a high-performance monochrome digital camera, Pixelink PL-A741 (1280×1204 pixels) was used. It has an IEEE1394 communication interface and its spectral range goes from 350 up to $1000 \mathrm{~nm}$. In the NIR range, the sensor module is a 
monochrome camera XEVA-FPA-2.5-320 (320×256 pixels) by Xenics with Camera Link interface and a spectral range that covers from 850 to $2500 \mathrm{~nm}$.

Once the system set-up was arranged, spectral and spatial calibration processes were performed before the acquisition of HIS images. These procedures are particularly relevant in the NIR range, because, few imaging spectroscopic systems based on a PGP device working in the NIR range were previously reported. The wavelength or spectral calibration defines the spectral axis. In the Vis-NIR range (García-Allende, 2007), it was performed using two different light sources, whose emission wavelengths were previously known: a laser at $670 \mathrm{~nm}$ and a $\mathrm{Hg}$-Ar lamp with multiple emission lines. To deal with the unavailability of such a multi-line light source in the NIR spectral range, a customdeveloped set-up consisting of a tuneable laser source in the L-band and two laser sources were employed (Garcia-Allende, 2008d). The axis alignment was performed by rotating the camera with respect to the spectrograph. The system is properly calibrated when a particular wavelength corresponds to the same pixel number at both sides of the spatial axis. The spatial calibration calculates the dimensions, length and width, of the observation line. Apart from the entrance slit of the ImSpector, it depends on the lens focal length and on the distance between the material and the lens. A pattern of uniformly distributed black and white lines were used to obtain the field of view of the system. Finally, and in a last step, the responsivity calibration is performed. Due to the non-linear spectral responses of the cameras, flat standard reflectance measurements do not result in flat curves. Thus, a reflectance calibration is required within the working spectral range. This is achieved through dark and white reference images. The dark image is obtained by blocking the light into the system, while the white reference comes from the reflectance measurement of Spectralon, a thermoplastic resin with a spectrally flat behaviour. Once the image sample is captured, it becomes compensated generating the calibrated one $R\left(\lambda_{i}, x_{i}\right)$ :

$$
R\left(\lambda_{i}, x_{i}\right)=\frac{S\left(\lambda_{i}, x_{i}\right)-D\left(\lambda_{i}, x_{i}\right)}{W\left(\lambda_{i}, x_{i}\right)-D\left(\lambda_{i}, x_{i}\right)}
$$

where $S\left(\lambda_{i}, x_{i}\right)$ is the sample value at wavelength $\lambda_{\mathrm{i}}$ and spatial position $x_{i}, D\left(\lambda_{i}, x_{i}\right)$ is the value for the dark reference and $W\left(\lambda_{i}, x_{i}\right)$ is the equivalent for the white reference. This calibration compensates offsets due to the CCD dark current, the light source colour temperature drift, and the lighting spatial non-uniformity across the scene line. In this way, the sample reflectance is extracted from the system response.

\section{From HIS measurements to significant information}

Once the diffuse reflectance is registered in the datacube, valuable automatic quality indicators must be generated. Accurate and real-time operation can only be accomplished if appropriate and intelligent criteria are implemented extracting relevant information from the data cube. The upper part of Figure 3 shows the two additional stages needed to get the desired quality indicators: data compression and data classification.

The "data compression" carries out the reduction of data volume. Too much not-relevant information could distract the classifier and decrease the identification accuracy. On the other hand, the loss of information if compression is excessive becomes another risk. A wide variety of methods (Workman \& Springsteen, 1998) are available. Basically, they are classified between (Figure 4): 
- "feature extraction" where the spectral data is projected into to a lower dimension space where only some features, or projections, are kept to represent the general information. PCA (Principal Component Analysis) and its variations are examples of this category (Moeslund, 2001-2002; Mignani et al., 2003; Koren et al., 2004; O’Farrell et al., 2005; Park \& Choi, 2004).

- "feature selection" methods that identify and ignore those variables that do not contribute to the classification. They work directly with the spectral data space providing advantages such as the interpretability of results. Sequential Floating Feature Selection (SFFS) algorithm (Ferri et al., 1994; Gomez-Chova, 2003) belongs to this category.

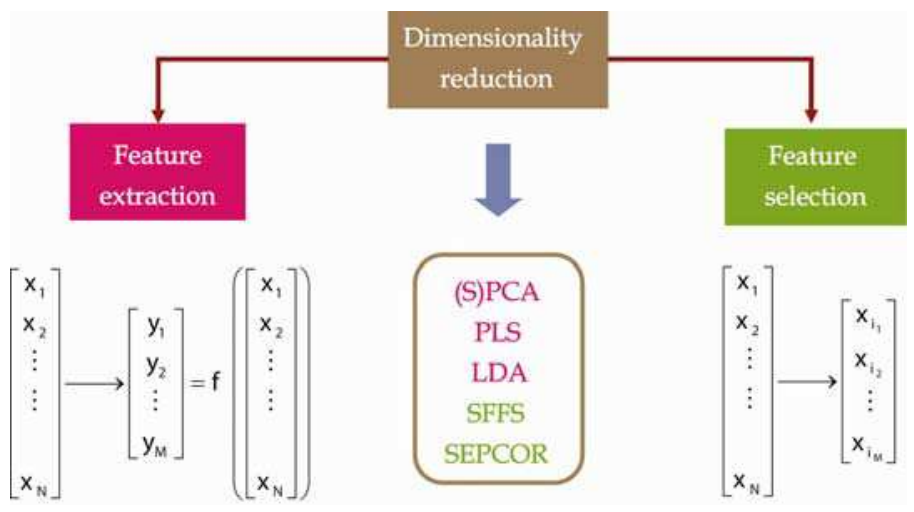

Fig. 4. Data compression alternatives: "feature extraction" and "feature selection"

\subsection{Feature extraction}

PCA is a feature extraction compression method that obtains the vectors or projection directions by maximizing the projected variance of a given data set. For dimensionality reduction purposes, the number of projection directions should be truncated trying to lose as less information as possible. PCA traditionally selects and filters vectors as a function of their corresponding eigenvalues. This approach is the so-called "m-method" (Moeslund, 2001) and, since it does not take into account any kind of class or category pertinence, it has several drawbacks. "J-measure" and "SEPCOR" (Moeslund, 2001) are also unsupervised methods like the "m-method". However, the first alternative selects those features that best separate the mean of the individual data categories and SEPCOR (SEPCOR, SEParability and CORrelation) takes into account both mean and variance of the classes, since the components are selected in decreasing order with respect to a variability measure:

$$
V\left(\vec{b}_{i}\right)=\frac{\text { Deviation of the class means }}{\text { Mean of the deviation of each class }}=\frac{\sum_{j=1}^{K}\left(\mu_{j i}-\mu_{i}\right)^{2}}{\sum_{g \in \text { clase } j}\left(y_{g i}-\mu_{j i}\right)^{2}}
$$

where $V\left(\vec{b}_{i}\right)$ is the variability of the $i^{\prime}$ th component, $\vec{b}_{i}$ is the $i^{\prime}$ th eigenvector, $K$ stands for the number of classes, $\mu_{\mathrm{ji}}$ is the mean of class $j$ in the input data at the $i^{\prime}$ th component and $y_{\mathrm{gi}}$ 
denotes a sample from the $g$ class in the $i$ 'th component. The numerator measures the distance between the class means and it would be desired to be large. On the other hand, the smaller the denominator, the better since it represents the compactness or clustering degree of the classes. Consequently, the larger the variability $V\left(\vec{b}_{i}\right)$ is, the better the component $\vec{b}_{i}$ behaves to discriminate classes. Section 6 will also compare, both qualitatively and quantitatively, the traditional approach ("m-method") with a supervised PCA alternative (SPCA, Supervised Principal Component Analysis). SPCA is not a different method for selecting eigenvectors. It is based on the modification of the original data set to include information related to the data class. This class information is considered afterwards for the projection (Chen, 2005).

Figure 5 represents the clustering degree obtained by the different PCA alternatives when spectra of tobacco leaves ( "target", green dots) are compared with spectra of spurious material ("non-target", red dots). "Non-target" spectra appear to be well dispersed due to the different chemical properties of this set of materials (wood, cardboard, leather, cellophane, other vegetable leaves, etc.). Figure 6 shows tobacco spectra only compared with diffuse reflectance spectra from other vegetable leaves in different maturity levels ("closestnon-target"). Although both groups remain still separated due to the specific chemical components of tobacco rich in alkaloids (nicotine, nornicotine, anabasine, etc.), sometimes they become mixed. This could induce identification errors in the classification stage. However, if the extracted PCA features become ordered following the variability criteria (2) (Conde, 2007) the enhancement in cluster separation is evident as shown in Figure 7, where SPCA exhibits a slightly better separation degree.

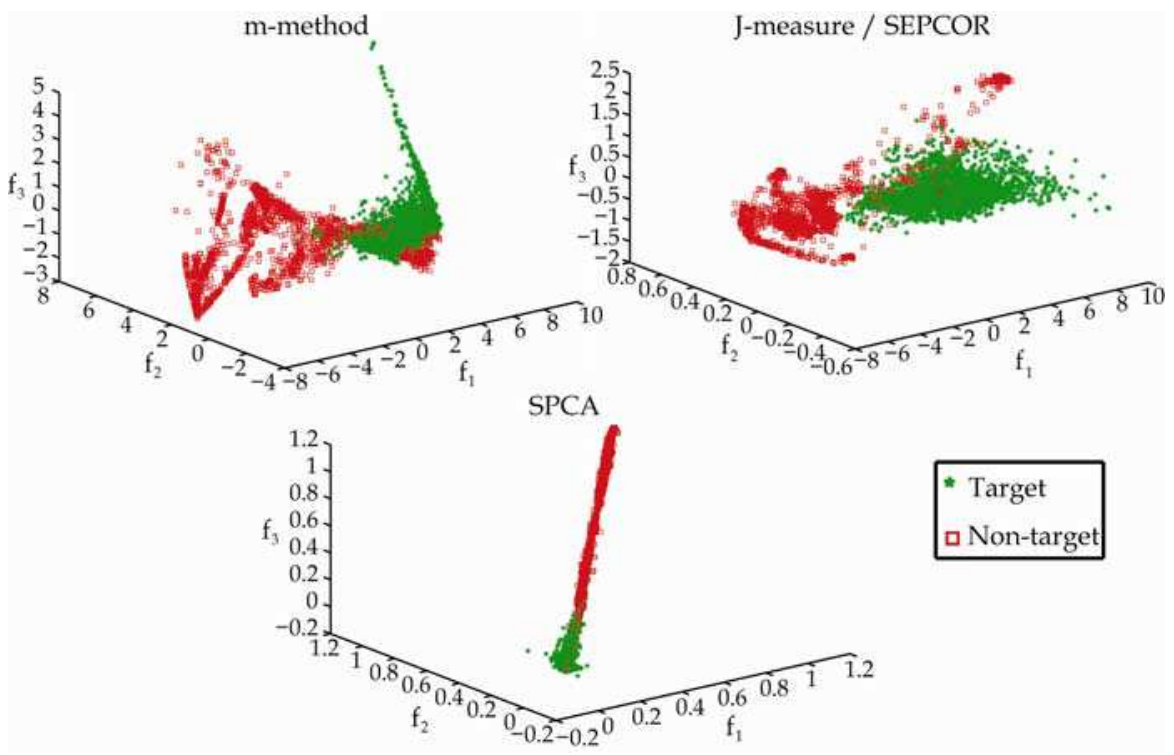

Fig. 5. Clustering of PCA alternatives for "target" material (tobacco leaves) and "non-target" material (all the spurious matter) 


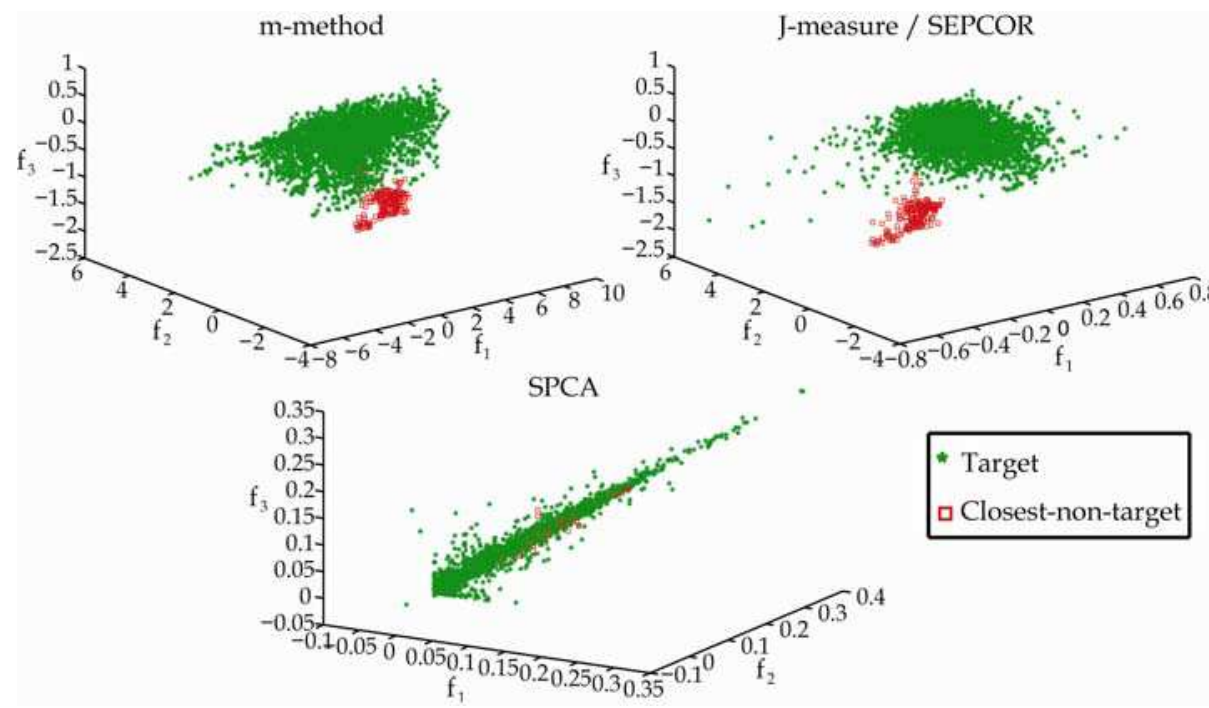

Fig. 6. Clustering of PCA alternatives for "target" material (tobacco leaves) and "closestnon-target" material (other vegetal spurious leaves)

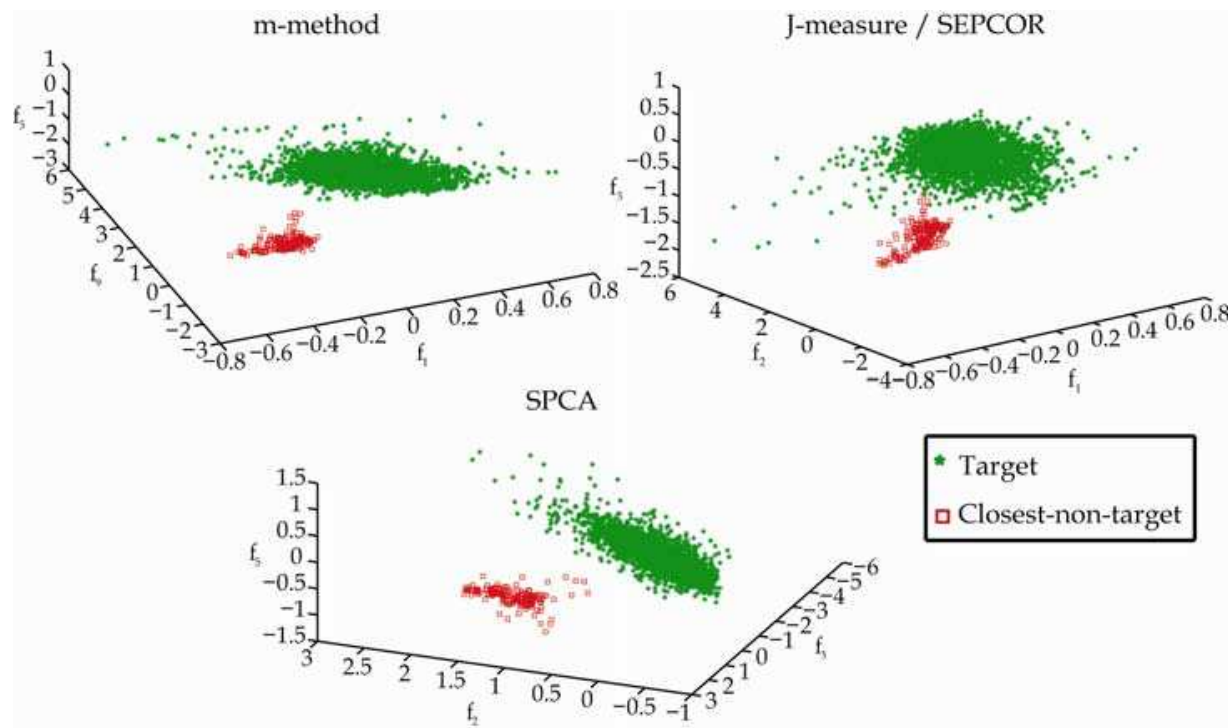

Fig. 7. Effect of variability ordering in PCA clustering for "target" material (tobacco leafes) and "closest-non-target" material (other vegetal spurious leaves) 


\subsection{Feature selection}

Feature selection methods come to solve some drawbacks of feature extraction techniques:

- When analyzing an unknown spectrum it is absolutely necessary to measure the intensity at all the spectral bands to be able to perform its projection.

- It is not possible to extrapolate the results to other spectrometers with different number of spectral bands.

- Result interpretation becomes a complex task as the extracted features cannot be associated with spectral bands with physical meaning.

Feature selection algorithms are first structured in terms of their selection criterion. "Class separability-based" algorithms assume that there are several classes, or statistical distributions, to be discriminated and choose those spectral bands that maximize the distance among classes in the lower dimensional space. On the other hand, those algorithms based on "classification performance" select the features that provide higher accuracy in subsequent classification stages. The latter is not so interesting because they have two major drawbacks. First, they have high computational loads, since it is necessary to train and test the classifier for each selected feature subset. Secondly, the obtained results cannot be extended to other classifiers.

The feature selection criterion is usually based on the calculation of a probabilistic distance. Chernoff, Bhattacharyya or Patrick-Fisher measurements are typically used. The Bhattacharya distance measures the class or category separability between the spectra of distinct materials, it is given by:

$$
J_{B}=\frac{1}{4}\left(\mu_{2}-\mu_{1}\right)^{T}\left[\Sigma_{1}+\Sigma_{2}\right]^{-1}\left(\mu_{2}-\mu_{1}\right)+\frac{1}{2} \ln \left(\frac{\left|\Sigma_{1}+\Sigma_{2}\right|}{2\left(\left|\Sigma_{1}\right| \cdot\left|\Sigma_{2}\right|\right)^{\frac{1}{2}}}\right)
$$

where $\mu_{i}$ and $\sum_{i}$ are the mean and the covariance matrix for class $i$. Gaussian distribution of the classes is assumed. In a multiclass discrimination problem, the separability measurement, $J$, would require to calculate the distance between every two classes:

$$
J=\sum_{i=1}^{C} \sum_{j=i+1}^{C} P_{i} P_{j} J_{i j}
$$

where $C$ is the number of classes, $P_{i}$ is each class probability and $J_{i j}$ is the distance between classes $i$ and $j$.

Apart from the selection criterion, an optimal search strategy of the feature bands must be followed because, even if the number of final selected features is known, the evaluation of the performance of each selected subset is unapproachable due to time consumption. A SFFS approach (Gomez-Chova, 2003) is interesting because it eliminates the redundant features after the addition of more useful ones and it also reconsiders the utility of some features that were possibly discarded before. Data decorrelation is compulsory prior to the application of SFFS. Blocks of correlated spectral bands are identified applying a threshold for the correlation coefficient, thus obtaining the correlation. Afterwards, only one wavelength of each block is selected. SFFS is then applied over this reduced set of wavelengths (Garcia-Allende, 2006; Garcia-Allende, 2009). 


\section{HIS classification alternatives for quality control}

After the discussion about how to obtain representative features of the material, "data classification" follows as depicted in Figure 3. Although data compression is essential, it is also compulsory the employment of an algorithm who identifies pixels whose spectrum exhibits a high degree of correlation ("matches"') to the expected material signature. Otherwise, the system will be useless in field applications. Several classification units can be implemented: Artificial Neural Networks (ANN), Fisher Linear Discriminant Analysis (FLD), Spectral Angle Mapper (SAM) and K-Nearest Neighbors (KNN). ANNs were initially employed because of their ability to handle non-linearity, their parallel processing of information and their quick adaptability to system dynamics (Nogueira, 2005). ANNs turned out to be very useful in this foreign object detection application and high classification accuracies (Garcia-Allende, 2007) were achieved, suggesting that maybe simpler algorithms could be employed instead. Consequently, classification units based on FLD and SAM were considered for spurious element detection because, due to their linearity, they become time efficient and then more appropriate for their employment in production lines of industrial environments. But with all of them, the addition of new products requires training again the system. To solve this, K-NN could be used instead (Fukunaga, 1990), given that no training is required for the classification. A brief introduction to these methods is presented in this section.

\subsection{SAM, Spectral Angle Mapper}

Spectral Angle Mapper is a simple algorithm based on the measurement of the spectral similarity between two spectra. This spectral similarity, $\alpha$, is obtained considering each spectrum as a vector in a $n$-dimensional space (Girouard et al., 2004):

$$
\alpha=\cos ^{-1}\left(\frac{\sum_{i=1}^{n} s_{1} s_{2}}{\left(\sum_{i=1}^{n} s_{1}^{2}\right)^{1 / 2} \cdot\left(\sum_{i=1}^{n} s_{2}^{2}\right)^{1 / 2}}\right)
$$

where $n$ is the number of spectral bands, and $s_{1}$ and $s_{2}$ are the two compared spectra. The angle between two spectra is therefore proportional to their similarity.

To implement a classification method based on SAM, two stages should be followed (Garcia-Allende, 2008f): training and test. In the training stage, reference spectra of the materials should be collected. For the wanted material a reference spectrum, $s_{w}$, is calculated by averaging homogeneous (the same kind of material in all the points of the line of vision) images. The unwanted material reference spectrum, $s_{u w}$, is determined by averaging images of the typical spurious materials in the selected application (foil, leather, plastics, etc.). In the test stage, an unknown image is classified. The spectral similarities $\alpha_{t-w}$ (between the spectrum of each spatial point, $s_{t}$, of the test image and $s_{w}$ ) and $\alpha_{t-u w}$ (between the test spectrum and $s_{u w}$ ) are calculated. If $\alpha_{t-w}<\alpha_{t-u w}, s_{t}$ is closer to $s_{w}$ and, therefore, that position contains tobacco. On the other hand, $\alpha_{t-u w}<\alpha_{t-w}$ indicates that $s_{t}$ corresponds to unwanted material. A schematic of the classification procedure is depicted in Figure 8, using a dimensionality of $n=3$ for visualization purposes. 


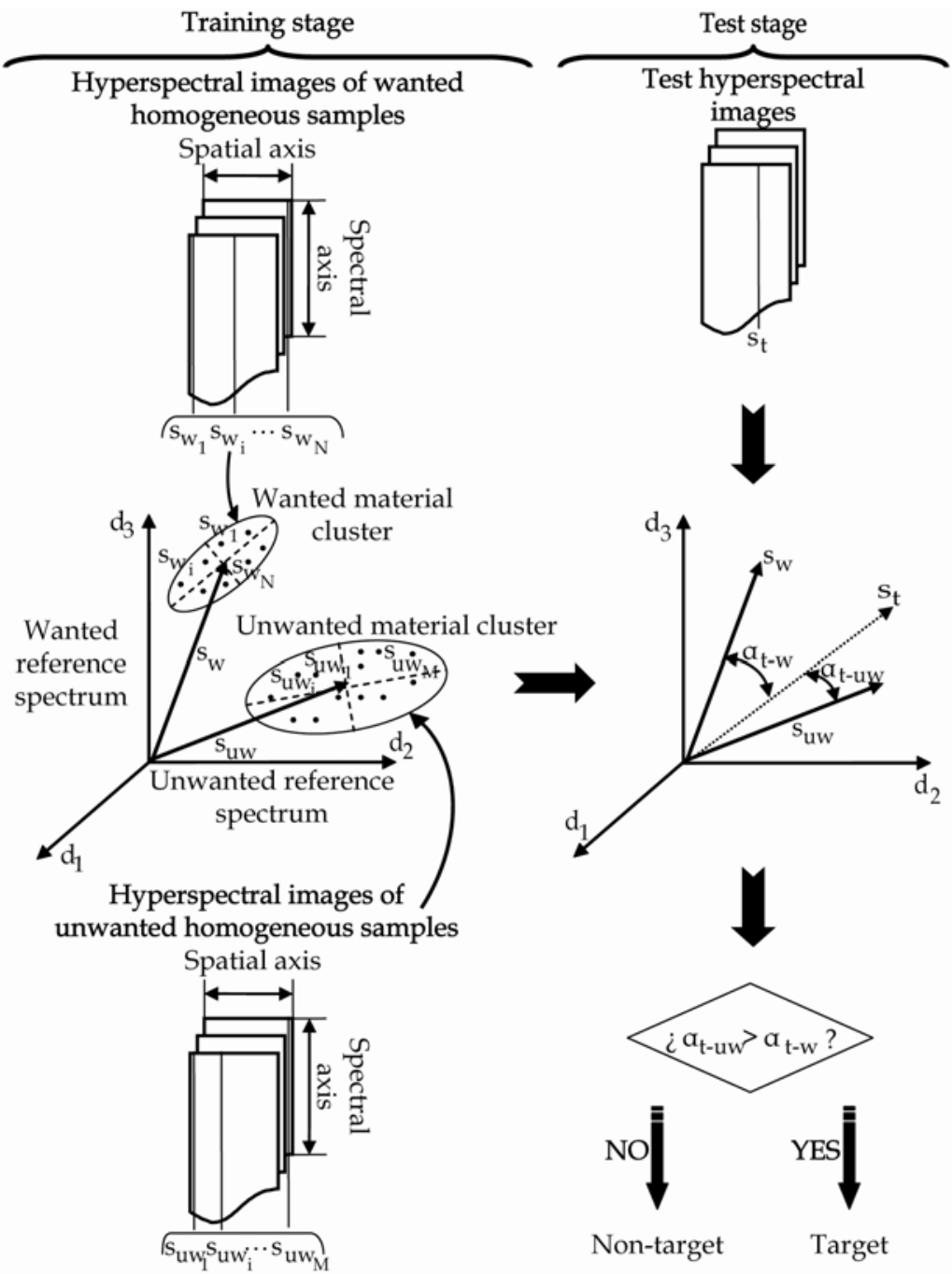

Fig. 8. SAM schematic description. "Training stage": calculation of the reference spectrum of each class $\left(s_{u w}\right.$ and $\left.s_{w}\right)$. "Test stage" an unknown spectrum $s_{t}$ is compared with these references for classification

\subsection{FLDA, Fisher Linear Discriminant Analysis}

This method projects high-dimensional data onto a lower-dimensional space in a way that best separates the classes in a least-squares sense. In the particular case of two material categories, the discriminant is one-dimensional and the direction that best discriminates the data is given by:

$$
w=S_{W}{ }^{-1}\left(m_{1}-m_{2}\right)
$$


where $S_{W}$ is the within-class scatter matrix, which is proportional to the sample covariance matrix, and $m_{1}$ and $m_{2}$ are the mean for each class. Then, each spectrum or data point, $r_{i}$, has a projection $y_{i}$ on $w$ given by

$$
y_{i}=w^{T} r_{i}
$$

The projections on $w$ of the two classes, "target" (wanted) and "non-target" (unwanted) are shown in Figure 9. For visualization purposes, each spatial point of the image is considered as a vector in a 3-dimensional space, where $d_{i}$ stands for each dimension.

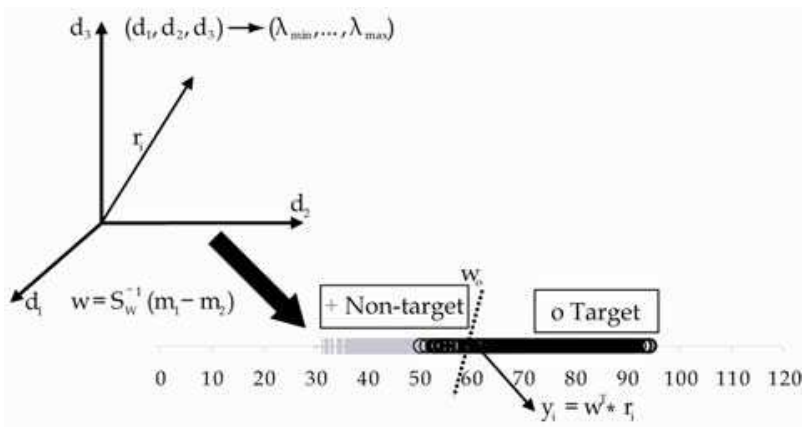

Fig. 9. Projections of the training set on the Fisher's linear discriminant

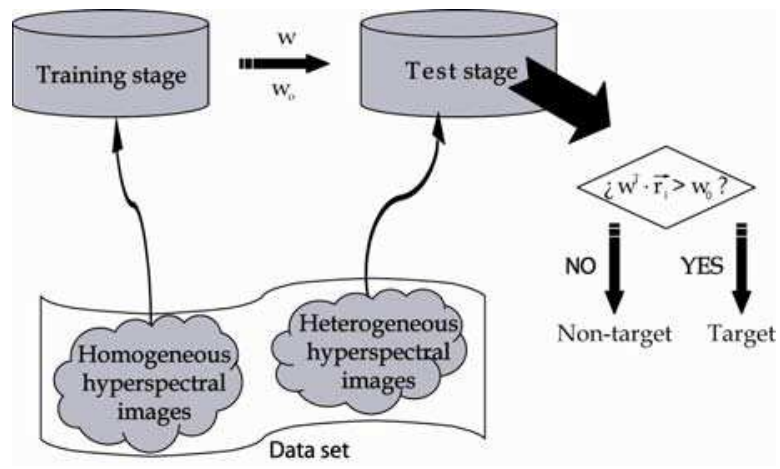

Fig. 10. Block diagram of image interpretation based on FLD

Since the discriminant is one dimensional, the projections of the material spectra on $w$ are numerical values. As a consequence, a threshold $w_{0}$ has to be fixed (Garcia-Allende, 2008e). When a material needs to be identified, its spectrum is projected onto $w$. Depending on its projection lies to the right or left of the decision threshold, $w_{0}$, the classification of the material will vary. The choice of the appropriate threshold is very important as it directly affects the classification error. Different strategies can be followed. The first one fixes the threshold, $w_{\text {str } 1}$, as the middle point of the means of each projected class. But, data variance could also taken into account, and the threshold $w_{\text {str } 2}$ could be determined as the middle point of the minimum of the "target" spectra projections and the maximum of the 
projections of "non-target" spectra. Figure 10 depicts a schematic description of the whole data processing system based on FLD.

$$
w_{s t r_{1}}=\frac{m_{2}+m_{1}}{2} \quad w_{s t r_{2}}=\frac{\left(m_{2}-\Delta_{2}\right)+\left(m_{1}+\Delta_{1}\right)}{2}
$$

\subsection{ANN, Artificial Neural Networks}

ANN have become really popular for their flexibility to different problems: chemical analysis (Eytan, 2000), arc welding (Garcia-Allende, 2008a), information technology (Messom, 2005), etc.

Neural networks emulate the human brain because they consist of layers of interconnected nodes. Each node produces a non-linear function of its input. The input to a node may come from other nodes or directly from the input data. Finally, some nodes are identified with the output of the network. The complete network, therefore, represents a very complex set of interdependencies which may incorporate any degree of nonlinearity, allowing very general functions to be modeled. There are multiple types of neural networks depending on the number of layers, the number of nodes per layer, transfer functions that are the transformation that each node produces of its input, training algorithm, etc. (Chen, 1996).

\subsection{K-NN, K-Nearest Neighbors}

The most significant advantage of a KNN classifier is that no training is required. When a new and unknown spectrum needs to be classified a comparison with the set of previously known spectra is performed. Consequently, KNN provides an enormous improvoment in foreign object detection applications in industrial scenarios because, since no training is required, new products to be discriminated can be added at any time.

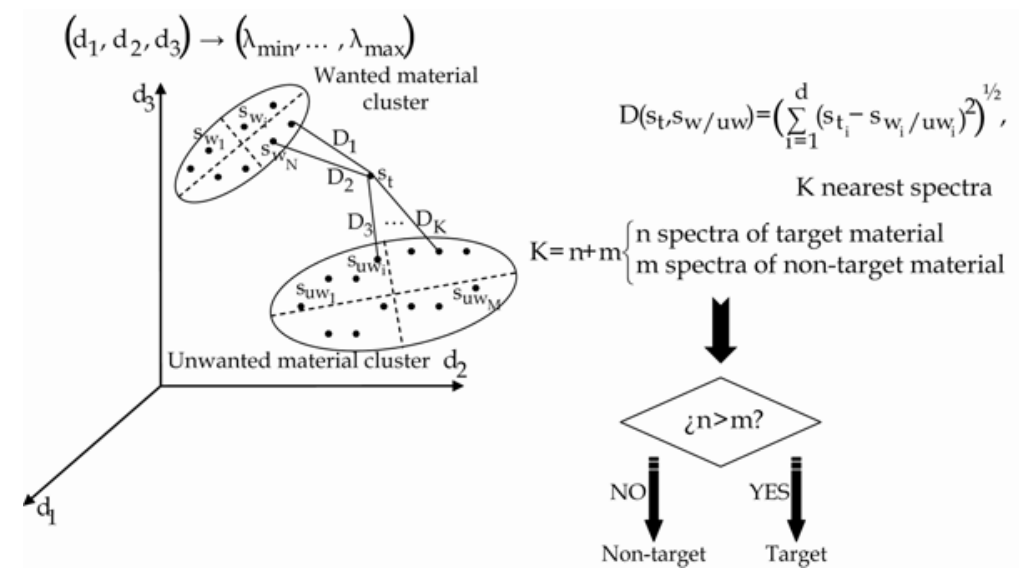

Fig. 11. KNN classifier where each feature is considered as a coordinate axis and the distance between points in this space determines how similar or different they are

A schematic of the KNN process for classifying the hyperspectral images is depicted in Figure 11 (Garcia-Allende, 2008g). The map is populated by points, each of which represents a spatial position of a particular image and as in previous cases. Since KNN is 
based on considering that similar data values should belong to the same material, then $K$ pixels with the most similar spectral properties to the unclassified spectrum are initially determined. This similarity is estimated in terms of the Euclidean distance. The unclassified pixel will be assigned to the most numerous class ("target" or "non-target") among the $K$ closest neighbors. Therefore, this classifier has only one independent parameter, $K$ or the number of neighbors, to consider.

\section{HIS performance evaluation for raw material sorting}

In essence, all considered methods are supervised algorithms where a training set of spectra is available with their corresponding materials labels. In the training dataset the expected classification outputs are one ("target") or zero ("non-target"'). For the test phase, "target" and "non-target" material are combined in the same line to confirm that the system is able to spatially discriminate them. A thorough and comprehensive work programme that included a wide variety of test samples was performed in this last stage. Figure 12 illustrates a couple of samples and their corresponding results. Its horizontal lines contain the following information:

- A photograph of the material sample under analysis. The white lines delimit the observation line where the horizontal axis corresponds to the spatial axis of the HIS image.

- $\quad$ A zoom of the observation line.

- The spectral images. The spatial axis is the horizontal one with the origin on the left as it appears in the real photograph. The spectral axis is the vertical one with the smallest wavelength of the spectral range at the bottom of the image.

- The results of the classification provided by the neural network when data dimensionality reduction is based on PCA (m-method).

- Idem but data compression is performed in this case by means of SFFS.

- Classification provided by the linear discriminant. The projection on $w$ of the reflectance of each spatial pixel of the observation line is also plotted in black. The decision threshold is plotted in green, showing those pixels of the observation line where the projection is higher than the threshold. These points are classified as "target" material.

- The last line shows SAM outputs.

Figure 13 shows a comparison between discrimination methodologies from a qualitative point of view. In order to accurately estimate the performance of the classification strategies, distinct cross-validation procedures should be applied. This way, the dependency of the results on the training or test sets is removed. Table 1 quantitatively compares different PCA algorithms in terms of the classification error attained by ANNs. A 3-fold cross-validation procedure (Zhu, 1996; Goutte-1997) was followed employing 11520 spectra from 120 images, 80 images of "target" material and 40 of "non-target" material. The ANN training-and-test procedure was repeated 3 times and the average values of success probability are displayed in Table 1. Although classification percentages are similar for all PCA methods, SPCA is confirmed as the best alternative. It is not possible to include also SFFS in this quantitative comparison in terms of the ANN classification indicators, because the network topology was not the same. Multilayer feed-forward networks with back-propagation learning algorithms were employed in both cases. The goal error considered in the training phase was $0.001 \%$. When data compression was performed by PCA methods, a network topology consisting of an input layer with 10 neurons and an output layer with one neuron was 
sufficient to achieve the training goal error (Garcia-Allende, 2007). When SFFS was implemented, the ANN only converged to the specified training error if the complexity of the topology was increased (a hidden layer with 5 neurons was included and the number of neurons in the input layer was reduced also to 5). A maximum classification accuracy of $94.62 \%$ was obtained by selecting either 10 or 12 spectral bands. Since compression rate is higher, 10 spectral bands would be selected in a practical implementation.

\begin{tabular}{|l|c|c|c|}
\hline $\begin{array}{c}\text { Selection } \\
\text { algorithm }\end{array}$ & $\begin{array}{c}\text { Number of } \\
\text { eigenvectors }\end{array}$ & $\begin{array}{c}\text { Processing time } \\
\text { [mseg] }\end{array}$ & $\begin{array}{c}\text { Success probability } \\
{[\%]}\end{array}$ \\
\hline m-method & 19 & 12 & 99.557 \\
\hline J-measure & 19 & 11 & 99.601 \\
\hline SEPCOR & 19 & 11 & 99.531 \\
\hline SPCA & 16 & 12 & 99.818 \\
\hline
\end{tabular}

Table 1. Quantitative comparison of dimensionality reduction for material sorting

Table 2 compares the classification capabilities of different strategies when the whole gallery of material samples was considered (again through a 3-fold cross validation procedure to attain generality on the training and test sets). Data compression was performed employing PCA ("m-method" discarding those eigenvectors whose associated eigenvalues are smaller than a thousandth of the maximum) in the case of classification units based on ANN and SAM, respectively. There is no need to use a pre-processing stage in the case of FLD because dimensionality reduction is directly obtained by the spectral projection onto the discriminant.

\begin{tabular}{|c|c|}
\hline Classification algorithm & Success Probability [\%] \\
\hline ANN & 99.557 \\
\hline FLD & 92.870 \\
\hline SAM & 90.540 \\
\hline
\end{tabular}

Table 2. Quantitative comparison of classification strategies

Test samples shown in Figure 12 and the success probability figures displayed in Tables 1 and 2 suggest that ANNs provide best classification performance than FLD and SAM. However, this does not imply that ANNs are better in a general sense. They only offer higher classification performance. FLD and SAM have, due to their linearity and simplicity, better time performance so, if identification errors are acceptable, they would be preferable. This situation resembles the actual one also in remote sensing. It has not been encountered yet, as pointed by Manolakis et al. (Manolakis et al., 2009), the best hyperspectral detection algorithm and it depends on the particular requirement applications. In the context of applications and experiences of quality control both, time consumption and difficulty for designers of hyperspectral imaging systems, should be taken into account to look through the existing literature. Consequently, the estimation of the expected level of performance of the variety of schemes presented throughout is a great asset for different future applications of imaging spectroscopy systems within the field. To aid in this selection, all the developed processing schemes are quantitatively compared in terms of the attained accuracy and their space complexity. The latter is the number of elementary objects that need to be stored during execution, i.e. the complexity of the hardware implementation compulsory to their employment in an embedded system in production lines. This comparison is presented in 
Figure 13 using a pseudo-color scale, where "green" corresponds to the best estimated performance. In a practical application, a trade-off has to be reached between accuracy and space complexity.

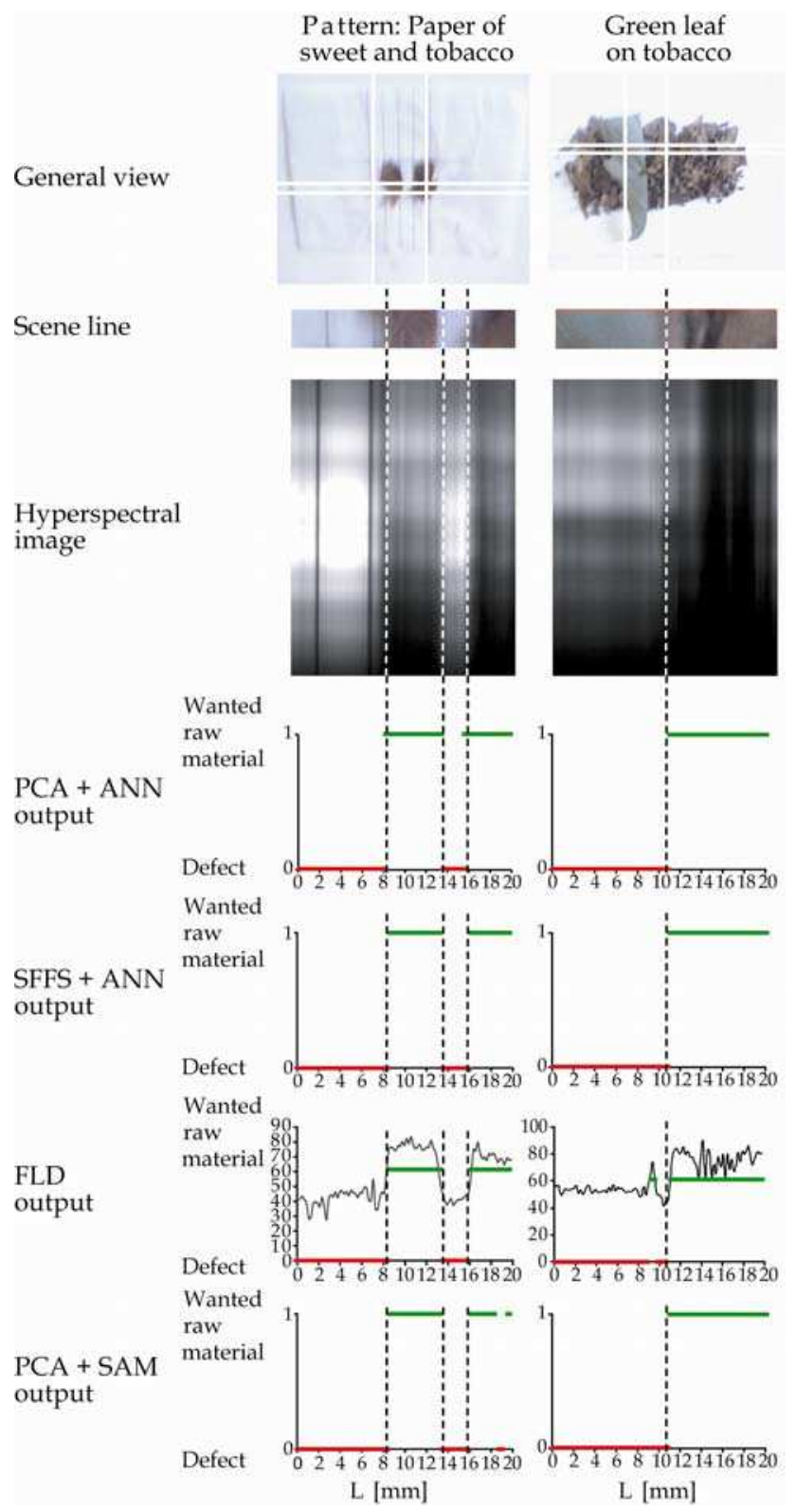

Fig. 12. Material classification results. Vertical dotted lines help to correlate discrimination outputs with the hyperspectral (third row) and visible (second row) images of material 


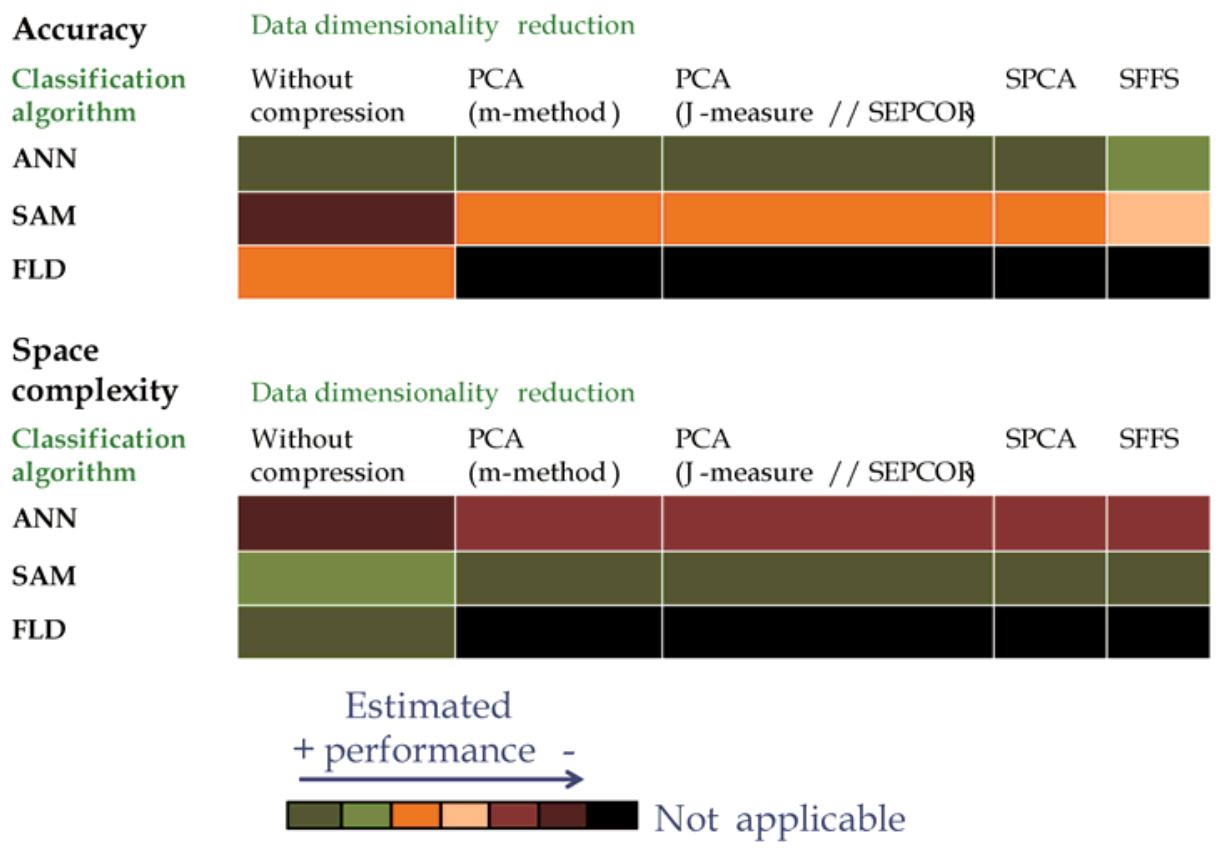

Fig. 13. Qualitative comparison of developed image processing strategies

\section{Conclusions}

In short, the main motivation of this chapter was to address two technological challenges that remained in HIS, i.e. the improvement of the actual acquisition systems and of the spectral interpretation methodologies. This improvements help to solve some issues in the niches focused on industrial applications, where high speed, in-line inspection and enhanced quality control are of primary concern. HIS systems on Vis-NIR and NIR spectral ranges have been designed, developed and validated. Special attention has been paid to calibration processes, wavelength and spatial calibration, since these procedures are particularly relevant in the NIR range. As shown in literature, HIS has gained prominence in recent years for solid material sorting.

The final output from the systems should be delivered in a straightforward way that facilitates its use. In raw material sorting, system usability, suggests that only the presence and position of spurious materials are of interest. Alternatives have been presented throughout this work related to the spectral interpretation of the HIS images to enhance the industrial usability of the technique. This includes proposed methodologies to achieve both dimensionality reduction and accurate determination of quality indicators. A variety of image analysis schemes were successfully validated in the tobacco industry. Different versions of PCA, both supervised (SPCA) and unsupervised ("m-method", "J-measure" and SEPCOR) were implemented to attain data compression. Their performances were qualitatively (clustering degree) and quantitatively (discrimination capability) compared. A feature selection method (SFFS) was also considered to reduce the data volume collected by 
the HIS system, and its advantages and drawbacks respect to feature extraction alternatives were specified and discussed. Finally, the sorting stage was addressed through different classification algorithms where their capabilities were also compared. A non-linear solution based on ANN was initially explored and very accurate spurious material detection was achieved with the appropriate training. This suggested that simpler algorithms could also be employed. Therefore classification units based on FLD and SAM were also proposed. Discrimination capability and time performances were compared but it was not possible, however, to conclude the superiority of any of them. While ANNs provided the best classification performance, FLD and SAM had better time behaviour. In some industrial contexts, probably FLD or SAM will be preferable if the provided sorting error is assumable. Some open research lines have been identified in the course of this work. A key challenge that remains in system design is that the scanning speed is tightly subject to the exposure time (several tenths of a second). Brighter sources would be required to perform more efficient measurements. A strong reduction in acquisition time could be achieved by the employment of high brightness supercontinuum sources (Alfano, 2006; Kaminski, 2008; Mignani, 2009). Additionally, studies on the possibility of using several light sources with narrower spectral ranges are ongoing. Specifically, an adaptive illumination source consisting of high-bright Light Emitting Diodes (LED) has been already envisaged (Conde, 2008) and applied with success to material sorting. Selected LED central wavelengths were those selected by the SFFS algorithm as the most discriminant of the spectral datacube.

Finally, a variety of interpretation/segmentation schemes of the acquired HIS images were proposed and all of them can be successfully extended to other sorting problems related to different materials.

Authors want to thank to M. Amado, F. Anabitarte, P. Anuarbe, A. Cobo for their contributions to this work.

\section{References}

Abbasgholipour, M.; Omid, M.; Keyhani, A. \& Mohtasebi, S.S. (2010). Color image segmentation with genetic algorithm in a raisin sorting system based on machine vision in variable conditions. Expert Systems with Applications, in press. 0957-4174.

Aikio, M. (2001). Hyperspectral prism-grating-prism imaging spectrograph, PhD Thesis, VTT Electronics.

Alfano, A.R. (2006). The Supercontinuum Laser Source, 2nd Ed, Springer, Berlin Heidelberg New York. 03-87245049.

Bonifazi, G. \& Serranti, S. (2006). Hyperspectral imaging based techniques in fluff characterization. Proc. of SPIE: Advanced environmental, chemical and biological sensing technologies $I V, 6377$, p. 637700. 0277-786X.

Bonifazi, G.; Serranti, S. \& D'Aniello, L. (2008). Compost quality control by hyperspectral imaging. Proc. of SPIE, 7003, p. 700321. 0277-786X.

Chen, W.H.; Hsu, S.H. \& Shen, H.P. (2005). Application of SVM and ANN for intrusion detection. Comput. Oper. Res., 32, 10, pp. 2617-2634. 0305-0548.

Chen. C.H. (1996). Fuzzy logic and neural network handbook. McGraw Hill. 978-0070111899.

Conde, O.M.; Amado, M.; Garcia-Allende, P.B.; Cobo, A.; Lopez- Higuera, J.M. (2007) Evaluation of PCA dimensionality reduction techniques in imaging spectroscopy for foreign object detection. Proc. of SPIE, 6565, p. 65650M. 0277-786X. 
Conde, O.M.; Cobo, A.; Cantero, P.; Conde, D.; Mirapeix, J., Cubillas, A.M. \& LopezHiguera, J.M. (2008). Adaptive illumination source for multispectral vision system applied to material discrimination. Proc. of SPIE, 7000, p. 70000Z. 0277-786X.

Eytan, O.; Sela, B.A. \& Katzir, A. (2000). Fiber-optic evanescent-wave spectroscopy and neural networks: application to chemical blood analysis. App. Opt., 39(19) pp. 33573360. 1559-128X.

Ferri, F.; Pudil, P.; Hatef, M. \& Kittler, J. (1995). Comparative study for techniques for large-scale feature selection in Pattern Recognition in Practice IV: Multiple Paradigms, Comparative Studies, and Hybrid Systems. E.S. Gelsema and L.N. Kanal

Fukunaga, K. (1990). Introduction to statistical pattern recognition. Academic Press, San Diego, $2^{\text {nd }}$ Ed. 0-12-2698517.

Garcia-Allende, P.B.; Conde, O.M.; Mirapeix, J.; Cubillas, A.M. \& Lopez-Higuera, J.M. (2006). Relevant Information Extraction from Hyperspectral Images for Quality Control Industrial Processes. ODIMAP V (5 th Topical Meeting on Optoelectronic Distance / Displacement, Measurements and Applications), pp. 271-276. 8-469009-38-9.

Garcia-Allende, P.B; Conde, O.M.; Cubillas, A.M.; Jauregui, C. \& Lopez-Higuera, J.M. (2007). New raw material discrimination system based on a spatial optical spectroscopy technique; Sensors and Actuators A: Physical, 135(2), pp. 605-612. 0924-4247.

García-Allende, P.B.; Mirapeix, J.; Conde, O.; Cobo, A. \& López-Higuera, J.M. (2008a). Arcwelding quality assurance by means of embedded fiber sensor and spectral processing combining feature selection and neural networks, Sensors, 8(10), pp. 6496-6506, 1424-8220.

Garcia-Allende, P.B.; Anabitarte, F.; Conde, O.M.; Madruga, F.J.; Lomer, M. \& LopezHiguera, J.M. (2008b). Infrared imaging spectroscopic system based on a PGP spectrograph and a monochrome infrared camera. Proc. of SPIE, 6941, p. 694118. 0277-786X.

Garcia-Allende, P.B.; Mirapeix, J.; Cobo, A.; Conde, O.M. \& Lopez-Higuera, J.M. (2008c). Arc welding quality monitoring by means of near infrared imaging spectroscopy. Proc. of SPIE. 6939, p. 69390Q. 0277-786X.

Garcia-Allende, P.B.; Conde, O.M.; Madruga, F.J., Cubillas, A.M. \& Lopez- Higuera, J.M. (2008d). Industrial defect discrimination applying infrared imaging spectroscopy and Artificial Neural Networks. Proc. of SPIE, 6939, p. 69390H. 0277-786X.

Garcia-Allende, P.B.; Conde, O.M.; Mirapeix, J.; Cobo, A. \& Lopez-Higuera, J.M. (2008e). Quality control of industrial processes by combining a hyperspectral sensor and Fisher's linear discriminant analysis. Sensors and Actuators B: Chemical, 129(2), pp. 977-984. 0925-4005.

Garcia-Allende, P.B.; Conde, O.M.; Mirapeix, J.; Cubillas, A.M. \& Lopez-Higuera, J.M. (2008f). Data Processing Method Applying Principal Component Analysis and Spectral Angle Mapper for Imaging Spectroscopic Sensors. IEEE Sensors Journal, 8(7), pp. 1310-1316. 1530-437X.

Garcia-Allende, P.B.; Conde, O.M.; Amado, M.; Quintela, A. \& Lopez-Higuera, J.M. (2008g). Hyperspectral data processing algorithm combining Principal Component Analysis and K Nearest Neighbours. Proc. of SPIE, 6966, p. 69660H. 0277-786X.

García-Allende, P.B.; Mirapeix, J.; Conde, O.; Cobo, A. \& López-Higuera, J.M. (2009). Defect detection in arc-welding processes by means of the line-to-continuum method and feature selection, Sensors, 9(10), pp. 7753-7770, 1424-8220. 
Girouard, G.; Bannari, A.; El Harti, A. \& Desrochers, A. (2004) Validated spectral angle mapper algorithm for geological mapping: comparative study between Quickbird and Landsat-TM. Geo-imagery bridging continents, Istanbul, July 12-23, pp. 599-604

Gomez-Chova, L.; Calpe, L.; Camps-Valls, G.; Martin, J.D.; Soria, E.; Vila, J.; Alonso-Corda, L. \& Moreno, J. (2003). Feature selection of hyperspectral data through local correlation and SFFS for crop classification. IEEE Int. Geosci. Remote Sens. Symp. Proc., 1, 555 - 557.

Gomez-Sanchis, J.; Gomez-Chova, L.; Aleixos, N.; Camps-Valls, G.; Montesinos-Herrero, C.; Molto, E. \& Blasco, J. (2008). Hyperspectral system for early detection of rottenness caused by Penicillium digitatum in mandarins. J. Food Eng., 89(1), pp.80 - 86. 02608774 .

Goutte C. (1997) Note on free lunches and cross validation. Neural Comput., 9(6), pp. 12451249. 0899-7667.

Green, R.O.; Eastwood, M.L.; Sarture, C.M. ; Chrien, T.G.; Aronsson, M.; Chippendale, B.J.; Faust, J.A. ; Pavri, B.E. ; Chovit, C.J. ; Solis, M.; Olah, M.R. \& Williams O. (1998). Imaging spectroscopy and Airborne Visible/Infrared Imaging Spectrometer (AVIRIS). Remote Sens. Environ., 65(3), pp. 227-248. 0034-4257.

Herrala, E.; Hyvarinen, T.; Voutilainen, O. \& Lammasniemi, J. (1997). An optoelectronic sensor system for industrial multipoint and imaging spectrometry. Sensor. Actuat. A-Phys., 61, 1-3, pp. 335 - 338. 0924-4247.

Kaminski, C.F.; Watt, R.S.; Elder, A.D.; Frank, J.H. \& Hult, J. (2008). Supercontinuum radiation for applications in chemical sensing and microscopy. Applied Physics B, 92, pp. 367-378. 0946-2171.

Koren, Y.; Tian, Q.; Zhou, X.S. \& Huang, T.S. (2004). Robust linear dimensionality reduction. IEEE T. Vis. Comput. Gr., 10, 4, pp. 459 - 470

Kotwaliwale, N.; Weckler, P.R.; Brusewitz, G.H.; Kranzler, G.A. \& Maness, N.O. (2007). Non-destructive quality determination of pecans using soft X-rays. Postharvest Biology and Technology, 45(3), pp. 372-380. 0925-5214.

Leitner, R.; McGunnigle, G.; Kraft, M.; de Biasio, M.; Rehrmann, V. \& Balthasar, D. (2009). Real-time detection of flame-retardant additives in polymers and polymer blends with NIR imaging spectroscopy. Proc. of SPIE, 7312, p. 73120M. 0277-786X.

Lu, R.; Chen, Y. R.; Park, B. (1999). Hyperspectral imaging for detecting bruises in apples. ASAE Annual International Meeting, Paper No. 99-3120.

Manolakis, D.; Lockwood, R.; Cooley, T. \& Jacobson, J. (2009). Is there a best hyperspectral detection algorithm? Proc. of SPIE, 7334, p. 733402. 0277-786X.

McClure, W.F.; Horris, K.H. \& Weeks, W.W. (1997). Rapid spectrophotometric analysis of the chemical composition of tobacco, Part 1: Total reducing sugars. BeitrÄage zur Tabakforschung International, 9(1), pp. 13 - 18.

Mesina, M.B.; de Jong, T.P.R. \& Dalmijn, W.L. (2007). Automatic sorting of scrap metals with a combined electromagnetic and dual energy X-ray transmission sensor. International Journal of Mineral Processing, 82(4), pp. 222 - 232. 0301-7516.

Messom, C.H.; Sarrafzadeh, A.; Johnson, M.J. \& Chao, M.J. (2005). Affective state estimation from facial images using neural networks and fuzzy logic in Neural networks: applications in information technology and web engineering. Borneo Publishing. 
Mignani, A.G.; Smith, P.R.; Ciaccheri, L.; Cimato, A. \& Graziano, S. (2003) Spectral nephelometry for making extravirgin olive oil fingerprints. Sensor. Actuat. B-Chem., 90(157), pp. 57 - 62. 0925-4005.

Mignani, A.G.; Ottevaere, H.; Ciaccheri, L.; Thienpont, H.; Cacciari, I.; Parriaux, O. \& Johnson, M. (2009). Innovative spectroscopy of liquids: a fiber optic supercontinuum source and an integrating cavity for scattering-free absorption measurements. Proc. of SPIE, 7503, p. 750377. 0277-786X.

Millar, S.J.; Whitworth, M.B.; Chau, A. \& Gilchrist, J.R. (2008). Mapping food composition using NIR hyperspectral imaging, New Food, 3, pp. 34-39.

Moeslund, T.B. (2001-2002) Principal component analysis. An introduction. Technical report, Aalborg University CVMT 01-02

Nogueira, A.; de Oliveira, M.R.; Salvador, P.; Valadas, R. \& Pacheco, A. (2005). Classification of internet users using discriminant analysis and neural networks. In NGI 2005: Next Generation Internet Networks: Traffic Engineering, pp. 341 - 348.

O'Farrell, M.; Lewis, E.; Lyons, W.B. \& Jackman, N. (2005). Combining principal component analysis with an arti ${ }^{-}$cial neural network to perform online quality assessment of food as it cooks in a large-scale industrial oven. Sensor. Actuat. B-Chem., 107, pp. 104 - 112. 0925-4005.

Park M.S. \& Choi, J.Y. (2004). Theoretical analysis on feature extraction capability of classaugmented PCA. Pattern Recogn., 42, pp. 2353-2362. 0031-3203.

Park, B.; Windham, W.R.; Lawrence, K.C. \& Smith D.P. (2004). Classification of hyperspectral imagery for identifying fecal and ingesta contaminants. Proc. of SPIE 5271, 1, pp. 118-127. 0277-766X.

Reich, G. (2005). Near-infrared spectroscopy and imaging: basic principles and pharmaceutical applications. Adv. Drug Deliver. Rev., 57(8), pp. 1109 - 1143. 0169409X.

Schmidt, W. (2005). Optical spectroscopy in chemistry and life sciences, Wiley-VCH, Weinheim. 978-3527299119.

Solo-Gabriele, H.M.; Townsend, T.G.; Hahn, D.W.; Moskal, T.M.; Hosein, N.; Jambeck, J. \& Jacobi, G. (2004). Evaluation of XRF and LIBS technologies for on-line sorting of CCA-treated wood waste. Waste Management, 24(4), pp. 413-424. 0956-053X.

Vaarala, T.; Aikio, A. \& Keraenen, H. (1997). An advanced prism-grating-prism imaging spectrograph in on-line industrial applications. Proc. of SPIE, 3101, pp. $322-330$. 0277-766X.

Varith, J.; Hyde, G.M.; Baritelle, A.L.; Fellman, J.K. \& Sattabongkot, T. (2003). Non-contact bruise detection in apples by thermal imaging. Innovative Food Science $\mathcal{E}$ Emerging Technologies, 4(2), pp. 211-218. 1466-8564.

Wiggenhauser, H.; Schaurich, D. \& Wilsch, G. (1998). LIBS for non-destructive testing of element distributions on surfaces. NDT E E International, 31 (4), pp. 307-313. $0963-$ 8695.

Willoughby, C.T.; Folkman, M.A. \& Figueroa M.A. (1996). Application of hyperspectral imaging spectrometer system to industrial inspection. Proc. of SPIE, 2599, pp. $264-$ 72. 0277-766X.

Workman Jr., J.; Springsteen, A.W. (1998) Applied Spectroscopy: A compact reference for Practitioners, London Academia Press Limited, 0127640703, London. 
Xing, J.; Bravo, C.; Jancsók, P.T.; Ramon, H. \& Baerdemaeker J. (2005). Detecting bruises on Golden Delicious apples using hyperspectral imaging with multiple wavebands. Biosystems Engineering, 90(1), pp. 27 - 36. 1537-5110.

Zhu, H. \& Rohwer, R. (1996). No free lunch for cross-validation. Neural Comput., 8(7), pp. 1421-1426. 0899-7667. 


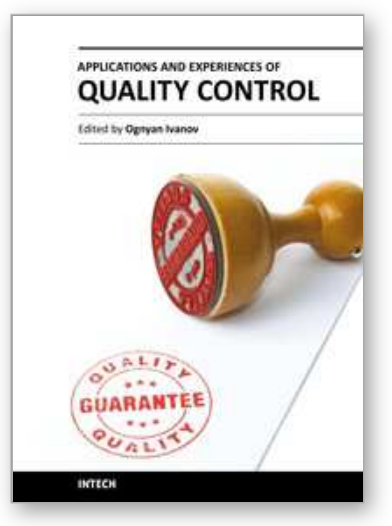

\section{Applications and Experiences of Quality Control \\ Edited by Prof. Ognyan Ivanov}

ISBN 978-953-307-236-4

Hard cover, 704 pages

Publisher InTech

Published online 26, April, 2011

Published in print edition April, 2011

The rich palette of topics set out in this book provides a sufficiently broad overview of the developments in the field of quality control. By providing detailed information on various aspects of quality control, this book can serve as a basis for starting interdisciplinary cooperation, which has increasingly become an integral part of scientific and applied research.

\section{How to reference}

In order to correctly reference this scholarly work, feel free to copy and paste the following:

Pilar-Beatriz García-Allende, Olga M. Conde and José Miguel López-Higuera (2011). Hyperspectral Imaging for Raw Material Sorting and Processed Product Quality Control, Applications and Experiences of Quality Control, Prof. Ognyan Ivanov (Ed.), ISBN: 978-953-307-236-4, InTech, Available from:

http://www.intechopen.com/books/applications-and-experiences-of-quality-control/hyperspectral-imaging-forraw-material-sorting-and-processed-product-quality-control

\section{INTECH}

open science | open minds

\author{
InTech Europe \\ University Campus STeP Ri \\ Slavka Krautzeka 83/A \\ 51000 Rijeka, Croatia \\ Phone: +385 (51) 770447 \\ Fax: +385 (51) 686166 \\ www.intechopen.com
}

\author{
InTech China \\ Unit 405, Office Block, Hotel Equatorial Shanghai \\ No.65, Yan An Road (West), Shanghai, 200040, China \\ 中国上海市延安西路65号上海国际贵都大饭店办公楼 405 单元 \\ Phone: +86-21-62489820 \\ Fax: $+86-21-62489821$
}


(C) 2011 The Author(s). Licensee IntechOpen. This chapter is distributed under the terms of the Creative Commons Attribution-NonCommercialShareAlike-3.0 License, which permits use, distribution and reproduction for non-commercial purposes, provided the original is properly cited and derivative works building on this content are distributed under the same license. 